\title{
LIPOPROTEINS AND THEIR SUBFRACTIONS IN PATIENTS WITH PSORIATIC ARTHRITIS: A 12-WEEK RANDOMIZED NUTRITIONAL INTERVENTION
}

Daniele Scherer ${ }^{1}$, Beatriz F. Leite ${ }^{1}$, Thauana L. Oliveira ${ }^{1}$, Rosana A. M. S. Freitas ${ }^{1}$, Nagila R. T. Damasceno¹, Marcelo M. Pinheiro ${ }^{1, \star}$

1.Universidade de São Paulo, São Paulo (SP), Brazil.

*Corresponding author: mpinheiro@uol.com.br

\section{BACKGROUND}

Atherogenic (Ath) and highly atherogenic (HAth) low-density lipoprotein (LDL) profiles are common in patients with psoriatic arthritis (PSA). Even patients with normal total cholesterol plasmatic levels can have a pro-atherogenic LDL pattern. A supervised and individualized diet intervention can improve lipoproteins profile, regardless omega 3 supplementation. To evaluate the impact of a nutritional intervention on plasma levels of high-density lipoprotein (HDL), LDL and their subfractions in patients with PsA.

\section{MATERIALS AND METHODS}

This was a randomized, placebo-controlled clinical trial of a 12-week nutritional intervention. Patients with PsA were randomly assigned to one of three groups: placebo, in which patients took $1 \mathrm{~g}$ of soybean oil daily, no dietetic intervention; diet + supplementation, in which patients followed an individualized diet, supplemented with $604 \mathrm{mg}$ of omega-3 fatty acids three times a day; and diet + placebo, supervised to an individualized nutritional adequacy $+1 \mathrm{~g}$ of soybean oil daily. The main outcomes were the Psoriasis Area and Severity Index (PASI) and disease activity score 28 (DAS28). The HDL and LDL subfractions were analysed by electrophoresis before and after the 12-week intervention. The LDL subfractions were classified as nonatherogenic (NAth), Ath or HAth, whereas the HDL subfractions were classified as small, medium or large particles. Values of $p<0.05$ were considered statistically significant.

\section{RESULTS}

Of the 91 patients evaluated, 56 (62\%) had an Ath or HAth profile. Thirty-two patients (35\%) had a high-risk lipoprotein profile despite having plasma LDL levels below $100 \mathrm{mg} / \mathrm{dL}$. Male gender, shorter disease duration and higher body mass index (BMI) were associated with the Ath and HAth profile. The 12-week nutritional intervention did not alter the LDL subfraction profile. However, there were significant increases in the HDL subfractions in all three groups.

\section{CONCLUSION}

Recognizing the pro-atherogenic subfractions LDL pattern could be a relevant strategy for identifying PsA patients with higher cardiovascular risk, regardless total LDL-cholesterol plasmatic levels. 